\title{
AN EVALUATION ON PRESCRIBING PATTERN OF ANTIBIOTICS IN PAEDIATRIC INPATIENTS AT TERTIARY CARE HOSPITAL
}

\author{
SABISHRUTHI S*, KAVITHA S, JAGAN NATHAN B, KALICHETI PRIYANKA, ARSHATH A \\ Department of Pharmacy Practice, Jaya College of Paramedical Sciences, College of Pharmacy, Chennai, Tamil Nadu, India. \\ Email: sabishruthi13@gmail.com
}

Received: 24 July 2019, Revised and Accepted: 10 October 2019

\section{ABSTRACT}

Objective: Our objective of the study was to evaluate the prescribing patterns of drugs with cost analysis in pediatric inpatients at tertiary care hospital.

Methods: It is an observational prospective study carried out in pediatric inpatients with a sample of 180 patients based on age, inclusion, and exclusion criteria for period of 3 months. The patient's data were collected using patient case record form and analysis of the data was done.

Results: Of 180 patients data were collected, the results show that majority of gender admitted in the hospital were male children 94 (52\%) and many are from age group of early childhood (2-5 years) 67 (37\%). Respiratory tract infections are diagnosis most commonly analyzed and off overall 236 prescribed antibiotics cephalosporins $86(43 \%)$ and combination of amoxicillin + clavulanic acid $25(71 \%)$ is the class of antibiotics prescribed higher than other class of drugs. Parental 173 (73\%) route of administration was observed to be followed more than other route. The percentage of cost variation of antibiotics observed in the study reveals that the huge variations were seen in the cost of medication in maximum and minimum cost.

Conclusion: The present study reveals that the prescribed antibiotics were as per the diagnosis of the patient and not by proceeding proper culture sensitivity testing. Hence, awareness about antibiotic must be created among practicing physicians to increase the therapeutic compliance of the patient.

Keywords: Pediatrics, Antibiotics, Prescribing, Culture sensitivity test, Awareness.

(C) 2019 The Authors. Published by Innovare Academic Sciences Pvt Ltd. This is an open access article under the CC BY license (http://creativecommons. org/licenses/by/4. 0/) DOI: http://dx.doi.org/10.22159/ajpcr.2019.v12i12.35074

\section{INTRODUCTION}

Antimicrobials are the most common drugs used in the treatment of various infections in the department of pediatrics [1]. Several antibiotics are prescribed unnecessarily in viral infection. Bacterial resistance is a risk for both community and individual which leads to fatal outcomes due to resistant for certain bacterial strains [2]. Inappropriate use of antibiotics results in antimicrobial resistance which leads to high risk in morbidity, mortality, and economic crisis on health care service [3]. In recent times, physicians are including antibiotics as an empirical therapy which may or may not be rational $[4,5]$.

Several studies have shown that antibiotics are most commonly prescribed in developed and developing countries for infections which cannot be avoided [2]. Infants and children are more prone to the infection and vulnerable to harmful effects of drugs due to differences in the pharmacodynamics and pharmacokinetics; hence, the ultimate goal is to accomplish rational use and cost effective [1]. Discriminate use of antibiotics leads to adverse drug reactions, suboptimal therapy, treatment failure, and polypharmacy [6,7]. Etiopathogenesis of the diseased condition and developmental stage of the child can be considered while prescribing at particular time. To minimize these problems and to assure safety antibiotics guidelines are required in the hospital setup $[4,7]$.

Antibiotics guidelines are standard guidelines developed based on culture sensitivity test for the treatment of infectious disease [7]. Evaluation of prescribing patterns helps in decreasing the adverse drug effect and to achieve the rational use of antibiotics. It helps to provide proper medical treatment of pediatric patients with accurate diagnosis, drug regimen, and rational use of antibiotics. Considering these case, the study aims to obtain the prescribing patterns of the antibiotics in the department of pediatrics.

\section{METHODS}

Study design and site

This is an observational and prospective study which was carried out in 500-bedded hospital. It was performed at pediatric department inpatient wards of tertiary care hospital from the duration of 3 months (April 2019-June 2019).

\section{Study population and sample}

Patients of both genders are included in the study. 180 patients were selected based on age, inclusion, and exclusion criteria. The wards were visited all 7 days of the week and patient's data were collected in a case record form.

Study criteria

Age criteria

\begin{tabular}{ll}
\hline Categories & Age groups \\
\hline Neonatal & Birth-12 months \\
Infant & 13 months-2 years \\
Early childhood & 2 years -5 years \\
Middle childhood & 6 years -12 years \\
\hline
\end{tabular}

Inclusion criteria

The following criteria were included in the study:

- Patients of either sex aged 0-12 years

- Patients admitted or treated to the department of pediatrics

- Patients caretakers who are willing to cooperate. 
Exclusion criteria

The following criteria were excluded from the study:

- Patients of either sex aged $>12$ years of age

- Patients who are not willing to cooperate

- Patients admitted in ICU.

Table 1: Gender of patients observed in the study $(n=180)$

\begin{tabular}{ll}
\hline Gender & Number of patients (\%) \\
\hline Male & $94(52)$ \\
Female & $86(48)$ \\
Total & $180(100)$ \\
\hline
\end{tabular}

Table 2: Age groups of patients observed in our study $(n=180)$

\begin{tabular}{ll}
\hline Age groups & Number of patients (\%) \\
\hline Birth-12 months & $25(14)$ \\
13 months-2 years & $30(17)$ \\
2 years-5 years & $67(37)$ \\
6 years-12 years & $58(32)$ \\
Total number of patients & $180(100)$ \\
\hline
\end{tabular}

Table 3: List of infections and systems affected to patients $(n=43)$

\begin{tabular}{ll}
\hline Types of systems and infections & Number of patients (\%) \\
\hline Respiratory tract infections & $11(26)$ \\
Dermatology & $7(16)$ \\
Gastrointestinal infections & $4(9)$ \\
Urinary tract infections & $1(2)$ \\
Central nervous system & $8(19)$ \\
Ear, nose, and throat & $3(7)$ \\
Cardiology & $2(5)$ \\
Hormonal disorders & $4(9)$ \\
Others & $3(7)$ \\
\hline
\end{tabular}

Table 4: Pharmacological classification of antibiotics prescribed to pediatrics $(n=201)$

\begin{tabular}{ll}
\hline List of class of antibiotics & Number of patients (\%) \\
\hline Macrolides & $27(13)$ \\
Fluoroquinolones & $14(7)$ \\
Nitroimidazoles & $13(6)$ \\
Penicillins & $18(9)$ \\
Cephalosporins & $86(43)$ \\
Sulphonamides & $1(1)$ \\
Anthranilic acid & $9(4)$ \\
Aminoglycosides & $23(11)$ \\
Carboxylic acid & $4(2)$ \\
Glycopeptides & $5(3)$ \\
Lincomycin & $1(1)$ \\
\hline
\end{tabular}

\section{Study materials}

Patient data collection form

Data were collected using a self-designed data collection form, which consists of details such as patient demographics, laboratory data, drug therapy, and other relevant information. Patient's demographic data contain the information of patient's name, age, sex, date of admission and discharge, chief complaints, diagnosis, history of patients, general and systemic examinations, details of prescribed medications, and their route of administration (ROA).

\section{Data analysis}

The prescriptions were analyzed for the numbers of antibiotics were prescribed, their dosage forms, frequency of the treatment, duration of the treatment was analyzed, and descriptive analysis is done. These data were analyzed using Microsoft Excel in the form of master sheet.

\section{Patient consent}

A self-designed patient consent form was used. Consent form prepared in both English and Tamil and consent was taken from patient guardian.

\section{RESULTS}

Gender of the patients

Of 180 patients, data were collected and the result shows that 94 (52\%) patients were male children and $86(48 \%)$ patients were female children. The numbers of male children were higher when compared to female children by $4 \%$.

\section{Age of the patients}

Of 180 patients data in the study from inpatient pediatrics department, the majority of the patients of early childhood 67 (37\%) belonged to age group of 2-5 years and the least patients were from neonates $25(14 \%)$ belonged to age group of birth -12 months.

List of infections and systems affected to the patients

Of overall 180 patients enlisted in the data, of 43 diagnosis, it was observed that respiratory tract infections in 11 (26\%), followed by central nervous system 8 (19\%), dermatological infections in 7 (16\%), gastrointestinal and hormonal disorders 4 (9\%), ear, nose, and throat and others $3(7 \%)$, cardiology $2(5 \%)$, and urinary tract infections $1(2 \%)$.

\section{List of classes of antibiotics prescribed to patients}

Of overall 180 patients enlisted in the data, it was observed that the class cephalosporins 86 (43\%) were prescribed higher than other classes of antibiotics, followed by macrolides 27 (13\%), aminoglycosides 23 (11\%), penicillins 18 (9\%), fluoroquinolones 14 (7\%), nitroimidazoles $13(6 \%)$, anthranilic acid $9(4 \%)$, glycopeptides $5(3 \%)$, sulphonamides, and lincomycin 1 (1\%).

\section{Combination of antibiotics prescribed in pediatric wards}

Of overall 180 patients enlisted in the data, it was observed that the combination of amoxicillin + clavulanic acid 25 (71\%) was prescribed more compared to other combinations, followed by cefadroxil + clavulanic acid $3(8 \%)$, paracetamol + mefenamic acid $2(6 \%)$, and other combinations $1(3 \%)$ were the least prescribed.

Table 5: Antibiotic combinations prescribed in pediatric wards $(n=35)$

\begin{tabular}{lll}
\hline Combination of antibiotics & Class of antibiotics & Number of patients (\%) \\
\hline Amoxicillin+clavulanic acid & Penicillin+beta lactamase inhibitor & $25(71)$ \\
Cefadroxil+clavulanic acid & Cephalosporin+beta lactamase inhibitor & $3(8)$ \\
Bacitracin+neomycin+polymyxin B & Bacitracin+aminoglycoside+polymyxin B & $1(3)$ \\
Paracetamol+mefenamic acid & Antipyretic+anthranilic acid & $2(6)$ \\
Ofloxacin+cefixime & Fluoroquinolones+cephalosporins & $1(3)$ \\
Cefpodoxime+clavulanic acid & Cephalosporins+beta lactamase inhibitor & $1(3)$ \\
Cefixime+linezolid & Cephalosporin+oxazolidione & $1(3)$ \\
Piperacillin+tazobactam & Penicillin+beta lactamase inhibitor & $1(3)$ \\
\hline
\end{tabular}




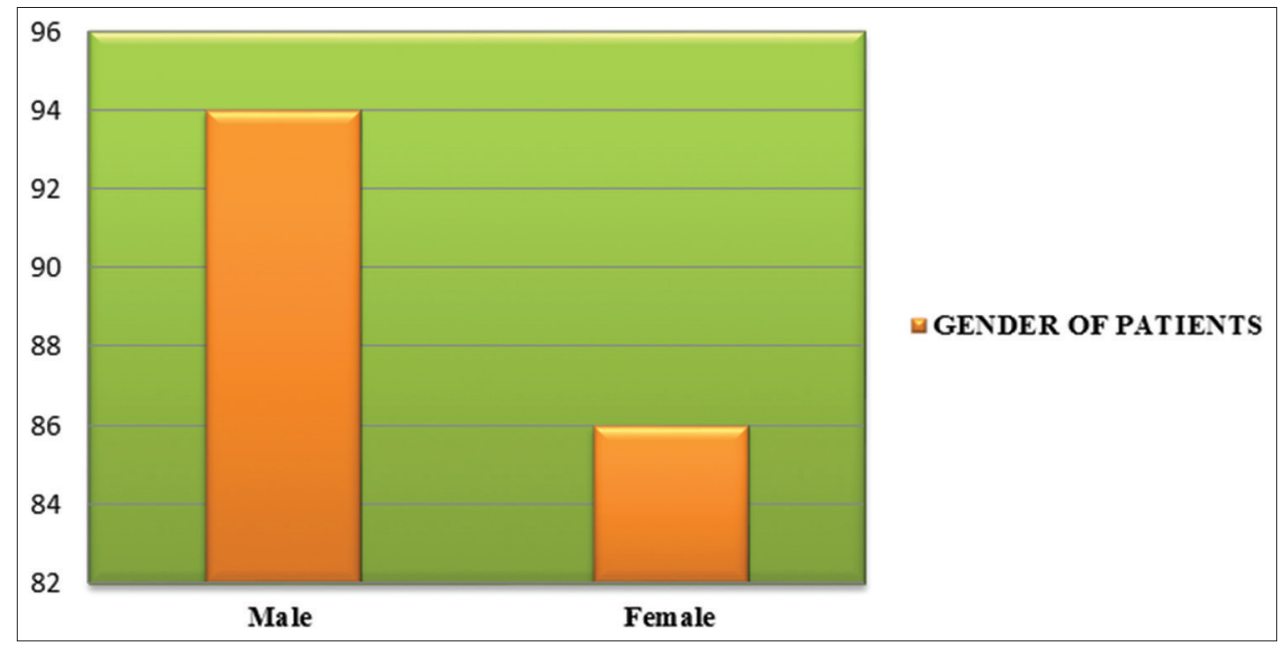

Fig. 1: Represents gender groups of patients

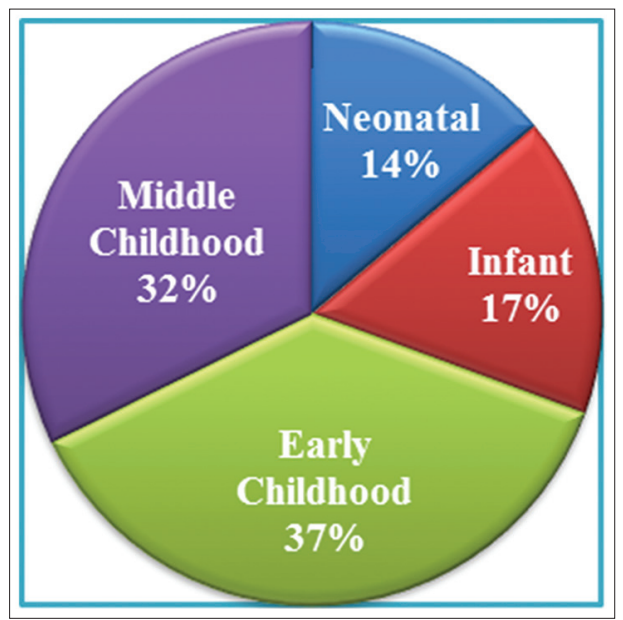

Fig. 2: Represents age groups of patients

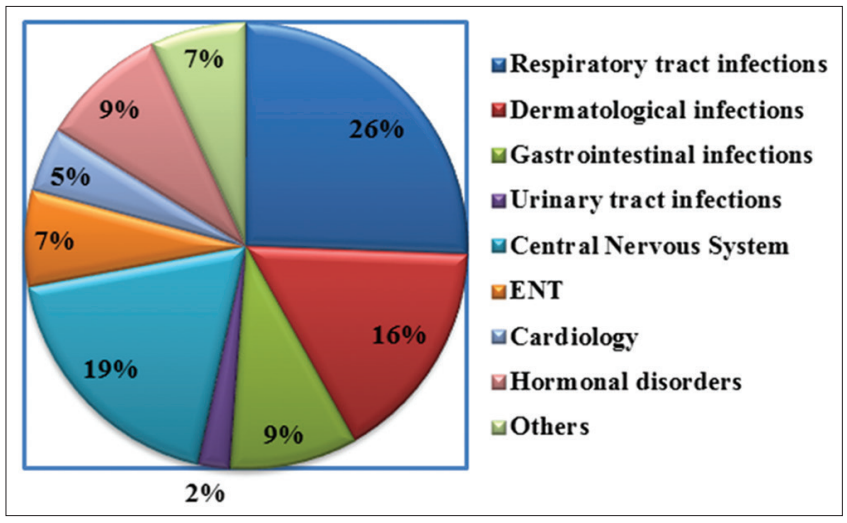

Fig. 3: The list of infections and systems affected to patients

Total number of antibiotics prescribed per patient

Of 180 prescriptions, the study reveals that the maximum number of prescriptions contains single antibiotics 135 (74\%) followed by multiple antibiotics prescribed per prescription.

\section{Frequency of antibiotics prescribed per antibiotics}

Of overall 236 antibiotic medications, 180 prescription study shows that twice daily $121(67 \%)$ are the most prescribed frequency of medications, followed by thrice daily $55(30 \%)$, once daily $3(2 \%)$, and least frequency prescribed was 4 times daily $1(1 \%)$ which was found in single prescription.

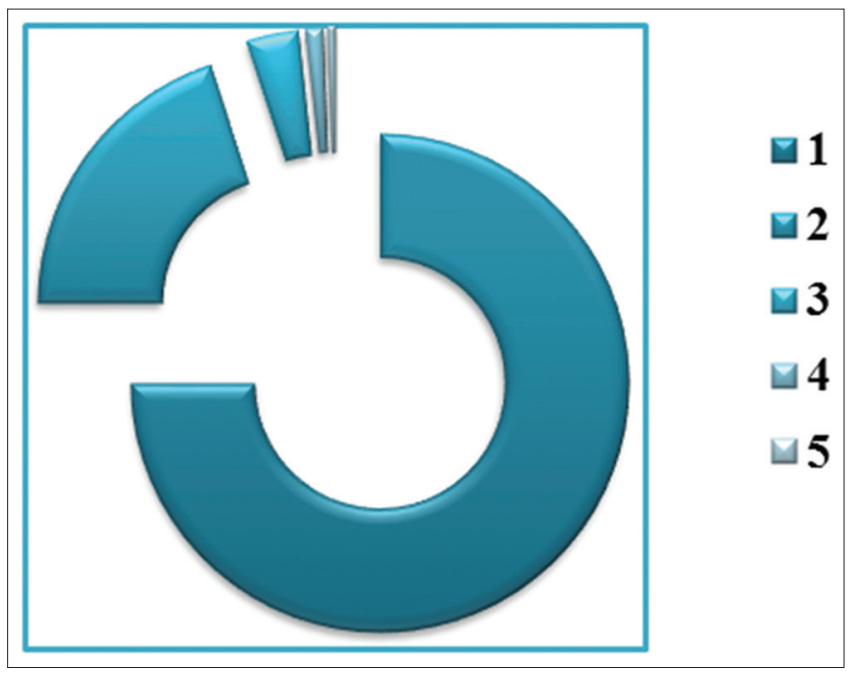

Fig. 4: Total number of antibiotics prescribed per patient

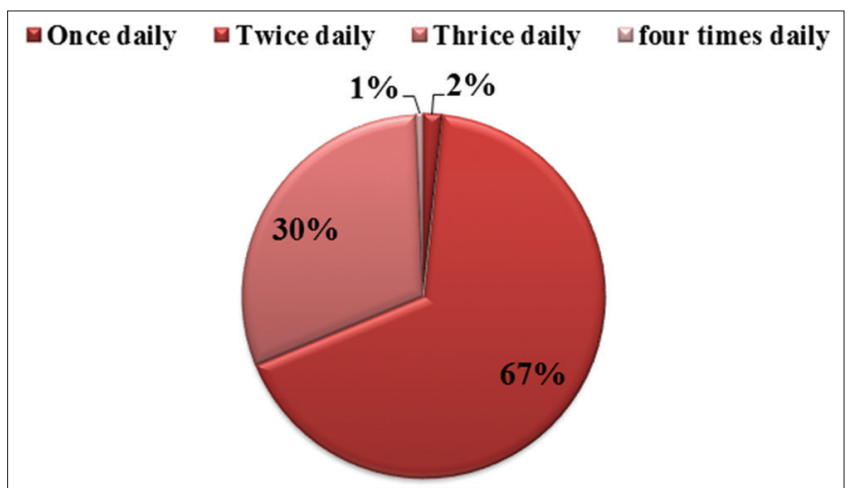

Fig. 5: Frequency of antibiotics prescribed per patients

ROA of drugs observed in the study

Of 236 drugs prescribed, the observation shows that most of the drugs administered through parental route $173(73 \%)$ both intravenous route and intradermal route followed by oral route $29(12 \%)$ and topical route $4(2 \%)$.

Cost variation of antibiotics observed in the study

The percentage of cost variation of antibiotics observed in the study reveals that the huge variations were seen in the cost of medication in maximum and minimum cost. Here from the average of cost, the maximum prices were seen within the cost of 0-100: 78 (33\%), followed 
by 600-700: 68 (29\%), 100-200: 30 (13\%), 700-800: 25 (11\%), and least average $1(0.4 \%)$ shows the cost more than 1000 .

\section{DISCUSSION}

The analysis reveals that the gender domination in our study was males (Table 1 and Fig. 1), which could be a common gender distribution showed in the majority of the studies and some study findings show similar results [8]. Most of the pediatrics were belongs to age distribution of 2-5 years (37\%) (Table 2 and Fig. 2); hence, these age group patients could be more susceptible for infections. Irrational use of antibiotics could result in poor therapeutic compliance of the patient. In present

Table 6: Total number of antibiotics prescribed per patient $(n=180)$

\begin{tabular}{ll}
\hline Number of antibiotics & Number of prescriptions (\%) \\
\hline 1 & $135(74)$ \\
2 & $36(20)$ \\
3 & $6(3)$ \\
4 & $2(2)$ \\
5 & $1(1)$ \\
Total & $180(100)$ \\
\hline
\end{tabular}

Table 7: Frequency of antibiotics prescribed to patients $(n=180)$

\begin{tabular}{ll}
\hline Frequency & Number of patients (\%) \\
\hline Once daily & $3(2)$ \\
Twice daily & $121(67)$ \\
Thrice daily & $55(30)$ \\
Four times daily & $11)$ \\
Total & $180(100)$ \\
\hline
\end{tabular}

study, percentage of more than two antibiotics prescribed is more and this indicates late diagnosis or selection of inappropriate antibiotic could be given without performed culture sensitivity testing. The majority of the antibiotic prescriptions were based on diagnosis (Table 3 and Fig. 3) of the patient, not on culture sensitivity test. Antibiotics

Table 8: ROA $(\mathrm{n}=236)$

\begin{tabular}{ll}
\hline ROA & Number of drugs (\%) \\
\hline Intravenous route & $117(50)$ \\
Oral route & $29(12)$ \\
Intradermal route & $86(36)$ \\
Topical route & $4(2)$ \\
Total & $236(100)$ \\
\hline
\end{tabular}

ROA: Route of administration

Table 9: Cost analysis of antibiotics observed in the study $(n=236)$

\begin{tabular}{ll}
\hline Cost & Number of antibiotics (\%) \\
\hline $0-100$ & $78(33)$ \\
$100-200$ & $30(13)$ \\
$200-300$ & $8(3.3)$ \\
$300-400$ & $9(3.8)$ \\
$400-500$ & $5(2.1)$ \\
$500-600$ & $6(2.5)$ \\
$600-700$ & $68(29)$ \\
$700-800$ & $25(11)$ \\
$800-900$ & $2(0.8)$ \\
$900-1000$ & $3(1.2)$ \\
$1000-1100$ & $1(0.4)$ \\
$1100-1200$ & $1(0.4)$ \\
\hline
\end{tabular}

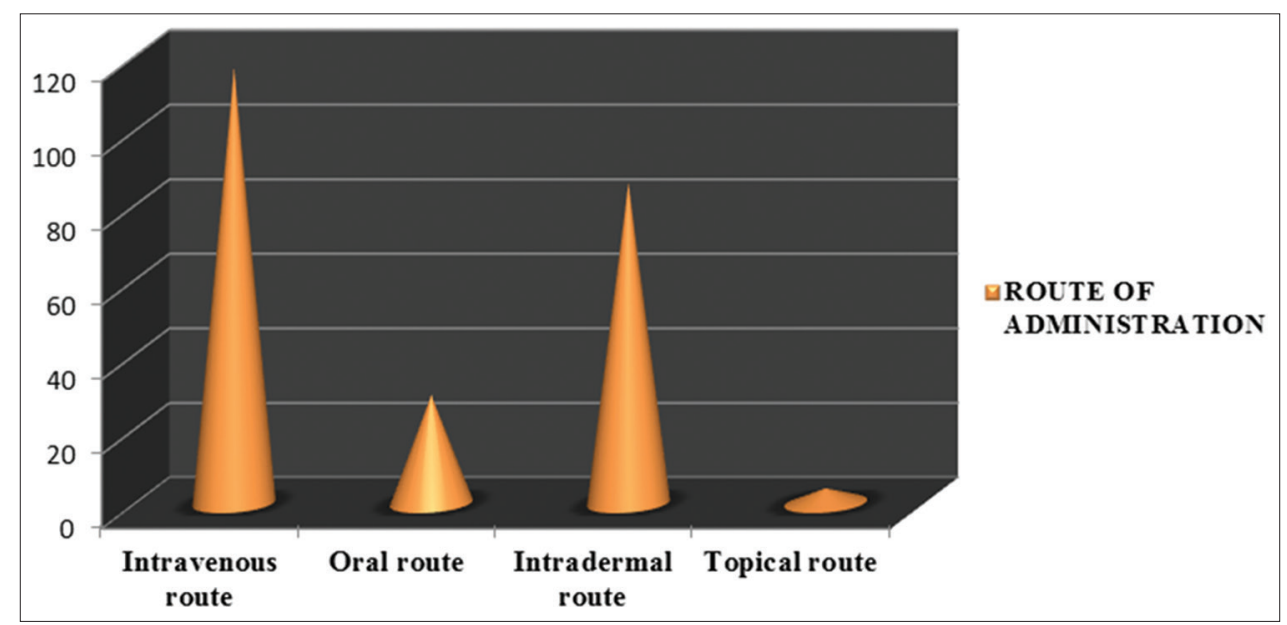

Fig. 6: The route of administration of drugs observed in the study

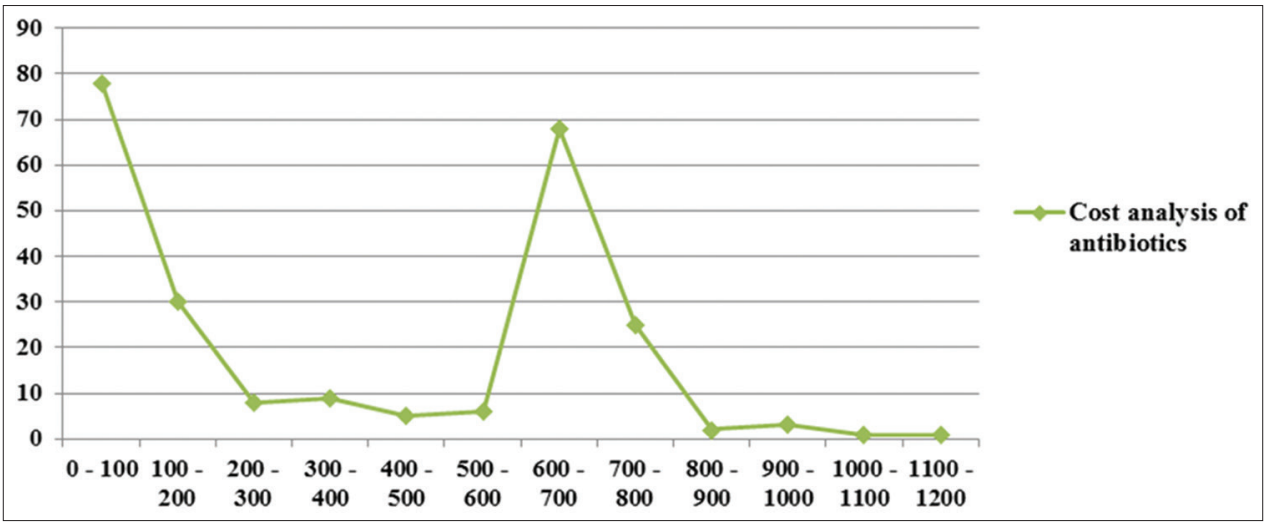

Fig. 7: The sketch line of cost variation observed in the study 
should be prescribed with proper examination. This helps to prevent antibiotic resistance, decreases the side effects/adverse effects of drugs and also reduces the cost of treatment to the patients [9-11]. Amoxicillin-clavulanic acid combinations (Table 5) were most commonly prescribed antibiotics, which could be easily available for the infectious conditions. Gupta et al. and Resi et al. study also reveal a similar result. Cephalosporins were most commonly prescribed medications (Table 4) in pediatrics for conditions such as respiratory tract infections and gastrointestinal infections [12,13]. Rad and Alekhya and de Bie et al. also show a similar result in his study $[14,15]$. The study shows that the maximum number of antibiotics prescribed was one followed by two and more than two (Table 6 and Fig. 4); the relavent result shows in Choudhury and Bezbaruah study [1]. Maximum frequency prescribed was twice a day (Table 7 and Fig. 5). Laya Vahdati Rad study also shows similar result [14]. In present study, $50 \%$ of antibiotics were administered by parenteral route (Table 8 and Fig. 6). As per WHO recommends lesser use of injection helps to decrease cost of the treatments which could be more helpful for patient from low socio economic background. Syrups are preferred instead of tablets or capsules for oral dosage regimens [16]. Costs of prescriptions were economic which indicated the irrational cost of antibiotics. About $29 \%$ of patients were prescribed at cost of Rs. 600-700 followed by 700-800. However, majority of the patients were prescribed below Rs. 100 (Table 9 and Fig. 7). Hence, prescribing pattern by keeping the number of medicines as low as possible, prescribing medicines by generic names, and using medicines appropriately after selecting will keep the cost of treatment as low as possible [17-20].

\section{CONCLUSION}

The present study reveals that the prescribed antibiotics were as per the diagnosis of the patient and not by proceeding proper culture sensitivity testing. Hence, this could also lead to development of antibiotic resistance in the patients. Following strict guidelines on antibiotics help to reduce the development of antibiotic resistance in children. The awareness about antibiotic must be created among practicing physicians to increase the therapeutic compliance of the patient. Physicians are advised to use generic names for prescribing drugs especially in pediatrics at private hospitals because to reduce the cost of therapy and to increase benefits of therapy.

\section{ACKNOWLEDGMENT}

The authors are thankful to Dr. C. Sekar, Chief Medical Superintendent, Government hospital, Thiruvallur, for his constant support and encouragement throughout the study. We thank the patients who gave their willing consent for the publication of their case by sharing complete information needed for the study.

\section{AUTHORSHIP CONTRIBUTION}

All the authors in the study have contributed their equal parts and comply with the journal authorship policy. Sabishruthi designed and framed the study; Kavitha and Jagan analyzed and interpreted the data and revised the article while Priyanka and Arshath participated in data collection and revision of article.

\section{COMPLIANCE WITH ETHICAL STANDARDS}

Written informed consent was obtained from the patient for publication. Copies of written consent may be requested for review from the corresponding author.

\section{CONFLICTS OF INTEREST}

The authors declare that they have no conflicts of interest concerning the content of this work.

\section{REFERENCES}

1. Choudhury DK, Bezbaruah BK. Antibiotic prescriptions pattern in paediatric inpatient department Gauhati medical college and hospital, Guwahati. J Appl Pharm Sci 2013;3:144-8.

2. Al-Ghazali MA, Alakhali KM, Alawdi S. Study of antibiotics prescribing pattern in paediatric patients of Thamar province, in republic of Yemen. J Appl Pharm 2017;9:1-4.

3. Pradeepkumar B, Alameri T, Narayana G, Reddy YP, Ramaiah JD. Assessment of antibiotic prescribing pattern in pediatric patients: A cross-sectional hospital-based survey. J Health Res 2017;4:235-7.

4. Malpani AK, Waggi M, Rajbhandari A, Kumar GA, Nikitha R, Chakravarthy AK. Study on prescribing pattern of antibiotics in a pediatric out-patient department in a tertiary care teaching and non-teaching hospital. Indian J Pharm Pract 2016;9:253-9.

5. Arnold SR, Allen UD, Al-Zahrani M, Tan DH, Wang EE. Antibiotic prescribing by pediatricians for respiratory tract infection in children. Clin Infect Dis 1999;29:312-7.

6. Zuppa A, Vijayakumar S, Jayaraman B, Patel D, Narayan M, Vijayakumar $\mathrm{K}$, et al. An informatics approach to assess pediatric pharmacotherapy: Design and implementation of a hospital drug utilization system. J Clin Pharmacol 2007;47:1172-80.

7. Kanish R, Gupta K, Juneja S, Bains H, Kaushal S. Prescribing pattern of antibiotics in the department of pediatrics in a tertiary care medical college hospital in Northern India. Asian J Med Sci 2014;5:69-72.

8. Narayan DS, Mangesh MM. A study of prescription pattern of antibiotics in paediatric in patients at a tertiary care hospital in central India. Int J Pharmacol Res 2016;6:286-90.

9. Leung E, Weil DE, Raviglione M, Nakatani H; World Health Organization World Health Day Antimicrobial Resistance Technical Working Group. The WHO policy package to combat antimicrobial resistance. Bull World Health Organ 2011;89:390-2.

10. Suman RK, Ray IM, Mohanty NC, Mukhia RK, Deshmukh YA. Assessment of usage of Antibiotic and their pattern of antibiotic sensitivity test among childhood fever. Int $\mathrm{J}$ Pharm Pharm Sci 2014;6:296-9.

11. Khanam US, Masud KN, Khurshed T, Chakma U. Antibiotics prescription pattern in rural area of Bangladesh: A cross-sectional study in Debidwar Upazila of Comilla district. Int J Pharm Pharm Sci 2018;10:36-40.

12. Gupta N, Safhi MM, Sumaily JM, Agarwal M. Drug prescribing patterns in children registered in the department of pediatrics of Jizan general hospital of Jizan, KSA. Int J Pharm Pharm Sci 2013;5:397-9.

13. Resi D, Milandri M, Moro ML, Romagna E. Antibiotic prescriptions in children. J Antimicrob Chemother 2003;52:282-6.

14. Rad LV, Alekhya M. Prescribing pattern of antibiotics in pediatric inpatient department of a tertiary care teaching hospital in Bangalore. IOSR J Pharm Biol Sci 2015;10:26-32.

15. de Bie S, Kaguelidou F, Verhamme KM, De Ridder M, Picelli G, Straus SM, et al. Using prescription patterns in primary care to derive new quality indicators for childhood community antibiotic prescribing. Pediatr Infect Dis J 2016;35:1317-23.

16. Piseh HN, Padwal SL, Jadhav RR, Deshmukh VS, Jadhav AD, Kolhe AM. Drug prescribing and dispensing pattern in pediatrics outpatient clinic of a rural tertiary-care teaching hospital. Natl J Physiol Pharm Pharmacol 2015;5:313-7.

17. Venkateswaramurthy N, Faisal EM, Sambathkumar R. Assessment of drug prescription pattern in paediatric patients. J Pharm Sci Res 2017;9:81-4

18. Jagan Nathan B, Sabishruthi S, Kavitha S, Sindhu S, Punitha S. An outlook on cost analysis among common oral antihypertensive medications of various brands available in Indian pharmaceutical companies. J Med Sci Clin Res 2019;7:89-95.

19. Purbandini CS, Sauriasari R. Cost-effectiveness analysis of ceftriaxone and non-ceftriaxone on typhoid fever patients. Int J App Pharm 2018;10:87-91.

20. Naveen V, Siddiq A, Chandana G. A study on drug utilization pattern of cephalosporins in general medicine and surgical inpatient department. Int J Curr Pharm Res 2018;10:33-6. 